\title{
O exercício físico modulando alterações hormonais em vias metabólicas dos tecidos musculoesquelético, hepático e hipotalâmico relacionado ao metabolismo energético e consumo alimentar The exercise modulating hormonal changes in metabolic pathways of skeletal muscle, liver and hypothalamus related to energy metabolism and food intake
}

Fábio Medici Lorenzeti*, Waldecir Paula Lima, D.Sc.**, Ricardo Zanuto, D.Sc.***, Luiz Carlos Carnevali Junior, D.Sc.***, Daniela Fojo Seixas Chaves, D.Sc. ${ }^{* * * *}$, Antônio Herbert Lancha Junior*****

*Técnico em Nutrição e Dietética (ETEC - Julio de Mesquita), membro do Laboratório de Nutrição e Metabolismo Aplicado a Atividade Motora - EEFE/USP, membro do Grupo de Estudos: Nutrição, Fisiologia e Treinamento - FEFISA, **Professor-Doutor do Instituto Federal de Educação, Ciência e Tecnologia de São Paulo - IFSP, membro do Laboratório de Metabolismo de Lipidios - ICB/USP, membro do Grupo de Estudos: Nutrição, Fisiologia e Treinamento - FEFISA, ${ }^{* * *}$ Laboratório de Sinalização Celular - ICB/USP, membro do Grupo de Estudos: Nutrição, Fisiologia e Treinamento - FEFISA, ****Membro do Laboratório de Metabolismo de Lipídios - ICB/USP, *****Professor titular da Universidade de São Paulo e coordenador do Laboratório de Nutrição e Metabolismo Aplicados à Atividade Motora (EEFE-USP)

\section{Resumo}

O exercício físico é responsável por gerar diversas adaptaçôes morfofuncionais, endócrinas, metabólicas e neurais. Dentre estas, destaca-se a melhora na sensibilidade à ação de hormônios como a insulina e a leptina, bem como a modulação nas concentrações plasmáticas dos hormônios GH, IGF-1, testosterona e cortisol, responsáveis pela homeostase energética. A insulina é um importante estimulante na secreção de leptina, ambos exercem papel central na homeostase energética e controle do consumo alimentar no núcleo arqueado do hipotálamo, controlando a secreção de neuropeptídios responsáveis pelo consumo alimentar, tais como: NPY, AgRP, CART e POMC. Esta revisão objetiva elucidar algumas ações do exercício físico relacionadas ao metabolismo e ao consumo alimentar, descrevendo algumas vias metabólicas que ocorrem nos tecidos musculoesquelético, hepático e, principalmente, hipotalâmico, ativadas por hormônios.

Palavras-chaves: exercício físico, hormônios, vias metabólicas, consumo alimentar.

\begin{abstract}
Exercise is responsible for generating various morphofunctional endocrine, metabolic and neural adaptations. Among them, there is the improvement in sensitivity to the action of hormones such as insulin and leptin, as well as modulation of plasma concentrations of hormones GH, IGF-1, testosterone and cortisol, responsible for energy homeostasis. Insulin is an important stimulation of leptin secretion, both have central role in energy homeostasis and control of food intake in arcuate nucleus of the hypothalamus, controlling the secretion of neuropeptides responsible for food intake, such as NPY, AgRP, POMC and CART. This review aimed to elucidate some of the actions related to exercise metabolism and food intake, describing some metabolic pathways that occur in skeletal muscle tissue, liver, and especially hypothalamic, activated by hormones.
\end{abstract}

Key-words: exercise, hormones, metabolic pathways, food consumption. 


\section{Introdução}

O exercício de média ou alta intensidade é responsável por gerar um balanço energético negativo [1,2]. Estudos longitudinais mostram indivíduos com perda de massa corporal em resposta à prática regular de um programa de exercícios físicos [3] .

Embora os procedimentos metabólicos na geraçáo de energia durante a prática de exercícios físicos justifiquem a manutenção da massa corporal, especula-se que esta prática possa contribuir para um equilíbrio energético e metabólico alterando a ingestáo de nutrientes [1].

Estudos com animais submetidos a diversos protocolos de treinamento físico apontam para uma melhora na sensibilidade à leptina em relação a animais controle sedentários [4-6] . É importante ressaltar que a leptina é responsável por inibir a secreção do neuropeptídeo Y (NPY) e estimular a secreção de POMC (pró-ópiomelanocortina) no núcleo arqueado do hipotálamo, responsáveis, respectivamente, por aumentar e inibir o consumo alimentar [7].

Diversos trabalhos apontam, também, que a prática de exercício físico promove o aumento de algumas citocinas, destacando-se as classes de interleucinas (ILs): IL-1, IL-6, IL-1 $\beta$ e IL-10 $[1,8]$. Destas, especula-se que o aumento de IL-6 relaciona-se ao aumento da atividade de 5’AMP proteína cinase ativada (AMPK) nos tecidos, adiposo e musculoesquelético. Contudo, no hipotálamo a IL-6 promove a diminuição da atividade de AMPK e acetil coenzima A carboxilase (ACC), além de ativar a via da mTOR (alvo de rapamicina em mamíferos), aumentando a fosforilaçáo das proteínas p70S6K (proteína ribossomal S6 cinase) e 4EBP1 (proteína de ligação do fator inicial de tradução eucariótico 4E), sendo responsável pelo controle da ingestão de nutrientes no hipotálamo [9].

A fosforilação de mTOR é uma importante via do controle da ingestão alimentar e homeostase energética, pois por meio da fosforilação da PI-3K (fosfoinositol 3 cinase) e da proteína cinase B (Akt) há o aumento da fosforilação da mTOR e das proteínas p70S6K ou 4EBP1; ressalta-se que esta via pode ser inibida pelas baixas concentraçôes plasmáticas de nutrientes como glicose e aminoácidos [1,9].

\section{Exercício de endurance e metabolismo}

O exercício físico de endurance é responsável por gerar diversas alteraçóes no metabolismo dos carboidratos, lipídios e das proteínas. O exercício físico aumenta a lipólise no tecido adiposo [10], principalmente pelo aumento nas concentraçôes plasmáticas de catecolaminas (adrenalina e noradrenalina) combinadas a diminuição nas concentraçóes de insulina, liberando ácidos graxos livres que serão captados pelo músculo durante o exercício. Sendo assim, o exercício físico é um importante modulador da secreção hormonal e da produção e consumo de energia [11].

A lipólise e a mobilização de ácidos graxos livres durante o exercício são influenciadas pelo estado nutricional, pela intensidade do exercício e pelo nível de condicionamento físico [10-13]. A contribuição total dos ácidos graxos livres para a produção energética é dependente do volume do exercício [10].

O exercício físico de endurance representa importante estimulo na modulação da expressão gênica dos receptores relacionados aos proliferadores de peroxissomas alfa, beta e gama (PPAR $\alpha$, PPAR $\beta$ e PPAR $\gamma$ ) [14-16]. A ativação do PPAR $\alpha$ e PPAR $\beta$ modula a expressão gênica de proteínas envolvidas nos processos de oxidação lipídica, tais como, piruvato desidrogenase cinase 4, malonil-CoA descarboxilase e carnitina palmitoil transferase-1 [16].

Já o PPAR $\gamma$ é responsável por modular proteínas relacionadas ao processo de lipogênese e lipólise nos adipócitos e hepatócitos respectivamente. Esta ação é exercida pela ativação de proteínas como a sintetase de ácidos graxos (tecido adiposo) e lipase hormônio sensível (fígado) $[17,18]$.

A secreção das IL-1 ra; IL-6 e IL-10 durante o exercício de endurance é responsável por inibir a ação do TNF- $\alpha$. Além de atuarem endocrinamente participando da liberaçáo de ácidos graxos pelo tecido adiposo para posterior oxidaçáo no músculo esquelético [19].

O músculo esquelético é capaz de captar glicose durante o exercício de endurance através de mecanismo que não utiliza a insulina como ativador do Glut-4, mas, sim, o cálcio que é liberado do retículo sarcoplasmático através da contração muscular [20].

O exercício físico de endurance é um importante ativador de 5'AMP proteína cinase ativada (AMPK). A AMPK é uma proteína heterodimérica ativada pelo estresse celular associado à depressão do ATP $[20,21]$. Sendo assim, ela é um importante sensor da quantidade de energia da célula, refletindo a relação entre AMP/ATP e creatina/fosfocreatina [20].

Desta forma, a ativação da AMPK no músculo esquelético é dependente da intensidade do exercício físico. A ativação da AMPK durante a contração muscular estimula a captação de glicose através da translocação do GLUT-4 [20,22].

Além disso, o exercício físico é responsável, também, por aumentar a taxa de difusáo da glicose para a célula muscular, visto que, após a sua captação a glicose é rapidamente fosforilada em glicose-6-fosfato pela ação enzimática da hexocinase [23-25].

\section{Exercício de endurance e consumo alimentar}

Diversas pesquisas procuram mostrar a relação entre o exercício físico de endurance e o consumo alimentar. Estudos realizados com ciclistas e maratonistas descrevem uma redução no consumo alimentar, por um fenômeno descrito como "anorexia induzida pelo exercício físico" [26-28]. Entretanto, a literatura afirma não haver alteraçóes crônicas geradas pelo exercício físico de endurance em relação ao aumento do consumo alimentar [29-31]. Ocorrendo então, apenas uma ação temporária do exercício sobre o consumo energético [2]. 
O efeito do exercício físico de endurance está diretamente relacionado com a secreção e ação hormonal. Dentre os hormônios envolvidos destacam-se a leptina, a grelina e a insulina (hormônio secretado pelas células $\beta$-pancreáticas). Entretanto, o exercício físico é capaz de modular a secreção de diversos hormônios como o cortisol, as catecolaminas (adrenalina e noradrenalina), $\mathrm{GH}$, hormônios tireoidianos $\left(\mathrm{T}_{3}\right.$ e $\left.\mathrm{T}_{4}\right)$ e os hormônios gonadais (testosterona e estrogênio), estes que por sua vez podem modular a secreção de leptina [31-33].

O exercício físico de endurance de alta intensidade é responsável por reduzir as concentraçôes plasmáticas tanto de insulina, quanto de leptina [33]. Entretanto, o exercício físico é capaz de modular positivamente a sensibilidade da açáo destes dois hormônios podendo ser este um dos motivos da anorexia induzida pelo exercício físico [34].

O estresse metabólico gerado pelo exercício físico de endurance de alta intensidade é responsável por aumentar a transcrição do gene da POMC pelos neurônios do núcleo arqueado do hipotálamo e pelos neurônios do trato solitário. A POMC por sua vez exerce seus efeitos biológicos através da interação com seu receptores de melanocortina 3 e 4 (MC3R e MC4R), levando a clivagem deste peptídeo e formação de outros peptídeos como ACTH e $\alpha$-MSH [35,36]. Este último age em neurônios do núcleo do trato solitário inibindo o consumo alimentar [37]. Entretanto, os mecanismos envolvidos nesta inibição ainda não estão totalmente elucidados [37].

A leptina é um hormônio constituído por 146 aminoácidos e secretado pelo tecido adiposo [32,38]. Atua no hipotálamo por meio do controle do balanço energético, ativando o sinal anorexígeno [39-41].

A secreção da leptina é oriunda do gene "ob" em resposta ao consumo alimentar, desencadeando assim um sinal anorexígeno em resposta ao aumento das concentraçôes plasmáticas de leptina [42].

Estudos apontam que a administração de leptina em ratos induz a uma menor expressão de neuropeptídeos ligados ao aumento do consumo alimentar $[43,44]$. Em contrapartida, o jejum diminui as concentraçôes plasmáticas de leptina, aumentando o consumo alimentar [42].

A leptina ao se ligar em seu receptor no hipotálamo (Ob$\mathrm{Rb}$ ) fosforila a proteína Janus cinase-2 (Jak-2), ativando a proteína STAT3 (em tirosina 705), promovendo a translocação dessa proteína para o núcleo se ligando ao DNA e ativando o fator transcricional SOCS3, gerando um feedback negativo na fosforilação da Jak2. Em função do feedback negativo, ocorre um cross-talk (refere-se a uma regulação cruzada entre uma determinada via metabólica sobre outra via metabólica. A este exemplo a ativação da via de sinalização intracelular da leptina, ativa paralelamente a via de sinalização da insulina) em relação às proteínas da cascata de sinalização da insulina IRS-1 e IRS-2 (substratos do receptor de insulina 1 e 2). $\mathrm{O}$ aumento da ativação da Akt gera sinalização intracelular inibindo o consumo alimentar e modulaçáo das ERKs, responsáveis pela homeostase energética [45].
A leptina é responsável, também, por reduzir a atividade de proteínas como AMPK e ativar a proteína alvo de rapamicina (mTOR) no hipotálamo [1], proteínas essas responsáveis pelo controle da ingestão alimentar no hipotálamo.

Trabalhos como o de Saladin et al. [46] referem que a insulina é um importante hormônio estimulante do gene ob e consequentemente estimulante da secreção de leptina.

Segundo Tups [47], o principal sinalizador da leptina no hipotálamo é a proteína PI-3k, que ativa Akt / PKB, por meio de um cross-talk, desencadeando assim o sinal anorexígeno.

A grelina é um hormônio constituído por 28 aminoácidos cuja secreção é feita pelas células estomacais em condições de balanço energético negativo, produzindo um sinal orexígeno - aumento do consumo alimentar - no hipotálamo [48,49].

Desta forma, a ação da grelina no hipotálamo é responsável por aumentar a secreção de neurotransmissores ligados ao aumento do consumo alimentar NPY e proteína relacionada ao agoti (AgPR) e diminuir a secreção de neurotransmissores ligados a restrição do consumo alimentar POMC e o fator de transcrito relacionado a cocaína e anfetamina (CART) $[48,49]$.

Para que a grelina esteja biologicamente ativa, esta deve ser acetilada no aminoácido serina pela ação da enzima Oaciltransferase (GOAT) [49]. Sendo assim, são encontradas no plasma a forma acetilada e a forma não acetilada ou desacetilada. Destas, a forma não acetilada encontra-se em maior concentração em relação à forma acetilada [50,51].

Estudos mostram que o exercício de endurance agudo é capaz de diminuir as concentraçôes plasmáticas de grelina acetilada. Contudo, o estudo de King et al. [52] avaliou a concentração de grelina total (acetilada e desacetilada) após doze semanas de treinamento de endurance $e$ não mostrou diferença estatística na quantidade de grelina acetilada entre o grupo treinado e o grupo controle.

\section{Exercício de força: metabolismo e consumo ali- mentar}

É bem estabelecido que o treinamento de força pode aumentar a área da secção transversa da fibra muscular, bem como trazer ganhos de força e potência [53,54]. Isso decorre das adaptaçóes neuromusculares promovidas pelo treinamento de força, especulando-se que com o estimulo mecânico há um aumento no número de RNAs mensageiros (mRNAs) envolvidos na síntese proteica no músculo esquelético e, também, na diminuição dos níveis de mRNAs de genes relacionados com o catabolismo muscular [55].

Trabalhos como o de Zanchi et al. [55] mostram que o treinamento de força é responsável por diminuir a expressão de genes como Atrogina-1 e MuRF-1, em relação a ratos sedentários, causando assim um aumento no ganho de força e aumento na área da secção transversa da fibra muscular.

Outra relaçáo com o treinamento de força é que ele é capaz de aumentar a expressão de proteínas como a proteína 
cinase B / Akt e a mTOR (alvo de rapamicina em mamíferos), responsáveis pela síntese proteica cursando com o aumento da massa muscular [56].

O músculo esquelético é responsável, não apenas pelas funçôes contráteis, mas, também, metabólicas do organismo humano, como metabolismo de aminoácidos, carboidratos e lipídeos, diminuindo a adiposidade e melhorando a sensibilidade a ação da insulina [56].

Os mecanismos de hipertrofia muscular, pelo treinamento de força, envolvem múltiplos fatores, tais como, estímulo mecânico, metabólicos, endócrinos e fatores neurais [53]. Estes fatores estão relacionados com a secreção de hormônios como GH (Hormônio de Crescimento), testosterona e IGF-1 (fator de crescimento semelhante à insulina-1) responsáveis pela resposta hormonal anabólica [57-61]. Ademais, estes hormônios modulam a secreção e ação de hormônios como insulina e leptina que podem atuar no núcleo arqueado do hipotálamo através dos mecanismos já descritos controlando o consumo alimentar e a homeostase energética.

É estabelecido que o IGF-1 exerce papel fundamental na regulação da glicemia e homeostase energética. Os mesmos autores relatam aumento nas concentraçôes plasmáticas de IGF-1 decorrentes do exercício e da alimentação [62].

Diferentemente do exercício físico de endurance, pouco se sabe a respeito das açôes do exercício de força em relação à homeostase energética e controle do consumo alimentar [63].

Em relação ao exercício físico de força, são bem conhecidos os mecanismos que envolvem o crescimento muscular por meio de uma complexa cascata de sinalização intracelular [58].

\section{Conclusão}

É bem estabelecido que o exercício físico modula as concentraçôes plasmáticas de diversos hormônios, dentre eles a insulina e a leptina. Estes hormônios representam importantes reguladores do consumo alimentar e da homeostase energética. Contudo, a cascata de sinalização destes hormônios envolve a ativaçáo de diversas proteínas-chave que podem modular a sensibilidade e levar aos seus efeitos biológicos finais. Tal cascata de sinalizaçáo é descrita em diversos trabalhos. Entretanto, todos os mecanismos que envolvem esta cascata ainda não estão totalmente elucidados, bem como o total efeito do exercício físico sobre o consumo alimentar.

Sendo assim, tornam-se necessários o desenvolvimento de mais estudos que investiguem a relação do exercício físico em diferentes intensidades e duração com estes hormônios reguladores da fome e saciedade, assim como os efeitos destes hormônios em relação a sua cascata de sinalização hipotalâmica.

\section{Agradecimentos}

Este trabalho foi apoiado por uma agencia brasileira de financiamento (FAPESP - Fundação de Amparo a Pesquisa do Estado de São Paulo, no 2010/08329-3).

\section{Referências}

1. Ropelle ER, Fernandes MF, Flores MB, Ueno M, Rocco S, Marin R, et al. Central exercise action increases the AMPK and mTOR response to leptin. PloS One 2008;3:e3856.

2. Martins C, Morgan L, Truby H. A review of the effects of exercise on appetite regulation: an obesity perspective. Int J Obes 2008;32:1337-47.

3. Wing RR, Hill JO. Successful weight loss maintenance. Annu Rev Nutr 2001;21:323-41.

4. Flores MB, Fernandes MF, Ropelle ER, Faria MC, Ueno M, Velloso LA et al. Exercise improves insulin and leptin sensitivity in hypothalamus of Wistar rats. Diabetes 2006;55:2554-61.

5. Heymsfield SB, Greenberg AS, Fujioka K, Dixon RM, Kushner $\mathrm{R}$, Hunt $\mathrm{T}$ et al. Recombinant leptin for weight loss in obese and lean adults: a randomized, controlled, dose-escalation trial. Jama 1999;282:1568-75.

6. Van Heek M, Compton DS, France CF, Tedesco RP, Fawzi $\mathrm{AB}$, Graziano MP et al. Diet-induced obese mice develop peripheral, but not central, resistance to leptin. J Clin Invest 1997;99:385-90.

7. Yee CL, Wang Y, Anderson S, Ekker M, Rubenstein JL. Arcuate nucleus expression of NKX2.1 and DLX and lineages expressing these transcription factors in neuropeptide $\mathrm{Y}(+)$, proopiomelanocortin(+), and tyrosine hydroxylase $(+)$ neurons in neonatal and adult mice. J Comp Neurol 2009;517:37-50.

8. Izquierdo M, Ibañez J, Calbet JA, Navarro-Amezqueta I, González-Izal M, Idoate F, et al. Cytokine and hormone responses to resistance training. Eur J Appl Physiol 2009;107:397-409.

9. Ropelle ER, Pauli JR, Fernandes MF, Rocco SA, Marin RM, Morari J, et al. A central role for neuronal AMP-activated proteinkinase (AMPK) and mammalian target of rapamycin (mTOR) in high-protein diet-induced weight loss. Diabetes 2008;57:594-605.

10. Stich V, Glisezinski I, Berlan M, Bulow J, Galitzky J, Harant I et al. Adipose tissue lipolysis is increased during a repeated bout of aerobic exercise. J Appl Physiol 2000;88:1277-283.

11. McMurray RG, Hackney AC. Interaction of metabolic hormones, adipose tissue and exercise. Sports Med 2005;35:393-412.

12. De Glisezinski I, Harant I, Crampes F, Trudeau F, Felez A, Cottet-Emard JM et al. Effect of carbohydrate ingestion on adipose tissue lipolysis during long exercise in trained men. J Appl Physiol 1998;84:1627-32.

13. Kempen KP, Saris WH, Senden JM, Menheere PP, Blaak EE, van Baak MA. Effect of energy restriction on acute adrenoreceptor and metabolic responses to exercise in obese subjects. Am J Physiol Endocrinol Metab 1994;267:E694-E701.

14. Russell AP, Feilchenfeldt J, Schreiber S, Praz M, Crettenand $A$, Gobelet $C$ et al. Endurance training in humans leads to fiber type-specific increases in levels of peroxisome proliferators-activated receptor- $\square$ in skeletal muscle. Diabetes 2003;52(2):874-81.

15. Tunstall RJ, Mehan KA, Wadley GD, Collier GR, Bonen A, Hargreaves $M$ et al. Exercise training increases lipid metabolism gene expression in human skeletal muscle. Am J Physiol Endocrinol Metab 2002;283: E66-72.

16. Muoio DM, MacLean PS, Lang DB, Li S, Houmard JA, Way JM et al. Fatty acid homeostasis and induction of lipid regulatory genes in skeletal muscle of peroxisome proliferatoractivated receptor (PPAR) $\nabla$ Knockout mice. Evidence for 
compensatory regulation by PPAR囚. J Biological Chemistry 2002;277(26):89-97.

17. Petridou A, Tsalouhidou S, Tsalis G. Long-term exercise increases de DNA binding activity of peroxisome proliferatoractivated receptor gamma in rat adipose tissue. Metabolism 2007;56(1):29-36.

18. Sugden MC, Zariwala MG, Holnes MJ. PPARs and orchestration of metabolic fuel selection. Pharmacol Res 2009;60:141150.

19. Petersen AMW, Pedersen BK. The anti-inflammatory effects of exercise. J Appl Physiol 2005;98(1):154-62.

20. Rose AJ, Richter EA. Skeletal muscle glucose uptake during exercise: how is it regulated? Physiology 2005;20:260-70.

21. Pereira LO, Lancha Junior AH. Effect of insulin and contraction upon glucose transport in skeletal muscle. Biophys Mol Biol 2004;84:1-27.

22. Barnes BR, Marklund S, Steiler TL, Walter M, Hjalm G, Amarger $\mathrm{V}$ et al. The 5-AMP-activated protein kinase 3 isoform has a key role in carbohydrate and lipid metabolism in glycolytic skeletal muscle. J Biol Chem 2004;279:38441-47.

23. King PA, Hirshman MF, Horton ED, Horton ES. Glucose transport in skeletal muscle membrane vesicle from control and exercise rats. Am J Physiol Cell Physiol 1989;257:C1128-C34.

24. Sakamoto K, Aschenbach WG, Hirshman MF, Goodyer LJ. Akt signaling in skeletal muscle: regulation by exercise and passive stretch. Am J Physiol Endocrinol Metab 2003;285:E1081E1088.

25. Zinker BA, Lacy DB, Bracy D, Jacobs J, Wasserman DH. Regulation of glucose uptake and metabolism by working muscle. An in vivo analysis. Diabetes 1993;42:956-65.

26. King NA, Blundell JE. High-fat foods overcome the energy expenditure induced by high-intensity cycling or running. Eur J Clin Nutr 1995;49:114-23.

27. Westerterp-Plantenga MS, Verwegen CR, Ijedema MJ, Wijckmans NE, Saris WH. Acute effects of exercise or sauna on appetite in obese and nonobese men. Physiol Behav 1997;62:1345-54.

28. Lluch A, King NA, Blundell JE. Exercise in dietary restrained women: no effect on energy intake but change in hedonic ratings. Eur J Clin Nutr 1998;52:300-7.

29. Thompson DA, Wolfe LA, Eikelboom R. Acute effects of exercise intensity on appetite in young men. Med Sci Sports Exerc 1988;20:222-27.

30. King NA, Snell L, Smith RD, Blundell JE. Effects of short-term exercise on appetite responses in unrestrained females. Eur J Clin Nutr 1996;50:663-67.

31. Blundell JE, King NA. Physical activity and regulation of food intake: current evidence. Med Sci Sports Exerc 1999;31:573-83.

32. Benatti FB, Lancha Junior AH. Leptina e exercício físico aeróbio: implicaçóes da adiposidade corporal e insulina. Rev Bras Med Esporte 2007;13:263-9.

33. Karamouzis I, Karamouziz M, Vrabas IS, Christoulas K, Kyriazis $\mathrm{N}$, Giannoulis E, et al. The effects of marathon swimming on serum leptin and plasma neuropeptide Y levels. Clin Chem Lab Med 2002;40:132-36.

34. Zoladz JA, Konturek SJ, Duda K, Majerczak J, Sliwowski Z, Grandys $M$ et al. Effect of moderate incremental exercise, performed in fed and fasted state on cardio-respiratory variables and leptin and ghrelin concentrations in young healthy men. J Physiol Pharmacol 2005;56:63-85.
35. Ellacott KL, Cone RD. The central melanocortin system and the integration of short- and longterm regulators of energy homeostasis. Recent Prog Horm Res 2204;59:395-408.

36. Fan W, Ellacott KL, Halatchev IG, Takahashi K, Yu P, Cone RD. Cholecystokinin-mediated suppression of feeding involves the brainstem melanocortin system. Nat Neurosci 2004;7:335-36.

37. Zheng H, Patterson LM, Rhodes CJ, Louis GW, Skibicka KP, Grill $\mathrm{HJ}$ et al. A potencial role for hypothalamo-medullary POMC projections in leptin-induced suppression of food intake. Am J Physiol Regul Integr Comp Physiol 2010;298:R7208.

38. Morash B, Li A, Murphy PR, Wilkinson M, Ur E. Leptin gene expression in the brain and pituitary gland. Endocrinology 1999;40:5995-8.

39. Halaas JL, Gajiwala KS, Maffei M, Cohen SL, Chait BT, Rabinowitz $\mathrm{D}$, et al. Weight-reducing effects of the plasma protein encoded by the obese gene. Science 1995;269:543-6.

40. Priego T, Sánchez J, Palou A, Picó C. Leptin intake during the suckling period improves the metabolic response of adipose tissue to a high-fat diet. Int J Obes 2010;1:809-19.

41. Maia-Monteiro CM, Bozza P. Leptin and mTOR partners in metabolism and inflammation. Cell Cycle 2008;7:1713-7.

42. Jéquier E. Leptin signaling, adiposity, and energy balance. Ann NY Acad Sci 2002;967:379-88.

43. Caron E, Sachot C, Prevot V, Bouret SG. Distribution of leptinsensitive cells in the postnatal and adult mouse brain. J Comp Neurol 2009;518:459-76.

44. Campfield LA, Smith FJ, Guisez Y, Devos R, Burn P. Recombinant mouse $\mathrm{OB}$ protein: evidence for a peripheral signal linking adiposity and central neural networks. Science 1995;269:546-9.

45. Burgos-Ramos E, Chowen JA, Argente J, Barrios V. Regional and temporal differences in leptin signaling in rat brain. Gen Comp Endocrinology 2010;167:143-52.

46. Saladin R, De Vos P, Guerre-Millo M, Leturque A, Girard J, Staels B et al. Transient increase in obese gene expression after food intake or insulin administration. Nature 1995;377:527-9

47. Tups A. Physiological models of leptin resistance. J Neuroendocrinol 2009;21:961-71.

48. Toshinai K, Date Y, Murakami N, Shimada M, Mondal MS, Shimbara T et al. Ghrelin-induced food intake is mediated via the orexin pathway. Endocrinology 2003;144:1506-12.

49. Kageyama H, Takenoya F, Shiba K, Shioda S. Neuronal circuits involving ghrelin in the hypothalamus-mediated regulation of feeding. Neuropeptides 2009;44:133-8.

50. Akamizu T, Takaya K, Irako T, Hosoda H, Teramukai S, Matsuyama A et al. Pharmacokinetics, safety, and endocrine and appetite effects of ghrelin administration in young healthy subjects. Eur J Endocrinol 2004;150:447-55.

51. Hosoda H, Doi K, Nagaya N, Okumura H, Nakagawa E, Enomoto $\mathrm{M}$ et al. Optimum collection and storage conditions for ghrelin measurements: octanoyl modification of ghrelin is rapidly hydrolyzed to desacyl ghrelin in blood samples. Clin Chem 2004;50:1077-80.

52. King NA, Gibbons CH, Martins C. Ghrelin and obestatin concentrations during puberty: relationships with adiposity, nutrition and physical activity. Med Sport Sci 2010;55:69-81.

53. Goto K, Ishii N, Kizuka T, Takamatsu K. The impact of metabolic stress on hormonal responses and muscular adaptations. Med Sci Sports Exerc 2005;36:955-63. 
54. Häkkinen K, Alen M, Kraemer WJ, Gorostiaga E, Izquierdo M, Rusko $\mathrm{H}$ et al. Neuromuscular adaptations during concurrent strength and endurance training versus strength training. Eur J Appl Physiol 2003;89:42-52.

55. Zanchi NE, Siqueira Filho MA, Lira FS, Rosa JC, Yamashita AS, Oliveira Carvalho CR et al. Chronic resistance training decreases MuRF-1 and Atrogin-1 gene expression but does not modify Akt, GSK-3beta and p70S6K levels in rats. Eur J Appl Physiol 2009;106:415-23.

56. Baar K, Nader G, Bodine S. Resistance exercise, muscle loading / unloading and the control of muscle mass. Essays Biochem 2006;42:61-74.

57. Holm L, Reitelseder S, Pedersen TG, Doessing S, Petersen SG, Flyvbjerg A et al. Changes in muscle size and MHC composition in response to resistance exercise with heavy and light loading intensity. J Appl Physiol 2008;105:1454-61.

58. Spiering BA, Kraemer WJ, Anderson JM, Armstrong LE, Nindl BC, Volek JS et al. Resistance exercise biology manipulation of resistance exercise programme variables determines the responses of cellular and molecular signalling pathways. Sports Med 2008;38:527-40.
59. Bosco C, Colli R, Bonomi R, von Duvillard SP, Viru A. Monitoring strength training: neuromuscular and hormonal profile. Med Sci Sports Exerc 2000;32:202-8.

60. Kraemer WJ, Marchitelli L, Gordon SE, Harman E, Dziados JE, Mello R et al. Hormonal and growth factor responses to heavy resistance exercise protocols. J Appl Physiol 1990;69:1442-50.

61. Mccall GE, Byrnes WC, Fleck SJ, Dickinson A, Kraemer WJ. Acute and chronic hormonal responses to resistance training designed to promote muscle hypertrophy. Can J Appl Physiol 1999;24:96-107.

62. Karl JP, Alemany JA, Koenig C, Kraemer WJ, Frystyk J, Flyvbjerg A et al. Diet, body composition, and physical fitness influences on IGF-I bioactivity in women. Growth Horm IGF Res 2009;19:491-6.

63. Ballard TP, Christopher LM, Camus H, Matthew C, Pitts J, Schmidt $S$ et al. Effect of resistance exercise, with or without carbohydrate supplementation, on plasma ghrelin concentrations and post-exercise hunger and food intake. Metabolism 2009;58:1191-9. 